\section{Prevention of mother-to-child transmission of hepatitis $B$ virus in Burkina Faso: Screening, vaccination and evaluation of post-vaccination antibodies against hepatitis B surface antigen in newborns}

Edwige T. Yelemkoure ${ }^{1,2}$

Albert T. Yonli, ${ }^{1,2}$ Carla Montesano, ${ }^{3}$

Abdoul Karim Ouattara, 1,2

Birama Diarra, 1,2

Théodora M. Zohoncon, $, 1,2$

Christelle W.M. Nadembega, ${ }^{1}$

Paul Ouedraogo, ${ }^{4}$

Charles Sombié, ${ }^{2}$ Serge Theophile

Soubeiga, ${ }^{1,2}$ Issoufou Tao, ${ }^{1}$

Adama Gansane, ${ }^{5}$ Massimo

Amicosante, ${ }^{3}$ Florencia Djigma, ${ }^{1,2}$

Dorcas Obiri-Yeboah, ${ }^{6}$ Virginio Pietra, ${ }^{2}$

Jacques Simpore, ${ }^{1-3}$ Vittorio Colizzi ${ }^{3}$

${ }^{1}$ Laboratory of Molecular Biology and

Genetics (LABIOGENE), UFR/SVT,

University Ouaga, Ouagadougou,

Burkina Faso; ${ }^{2}$ Biomolecular Research

Center Pietro Annigoni (CERBA),

Ouagadougou, Burkina Faso;

${ }^{3}$ Department of Biology, Tor Vergata

University of Rome, Italy; ${ }^{4}$ Saint

Camille Hospital of Ouagadougou

(HOSCO), Burkina Faso; ${ }^{5}$ Centre

National de Recherche et de Formation sur le Paludisme, Ouagadougou,

Burkina Faso; ${ }^{6}$ Department of

Microbiology and Immunology, School

of Medical Sciences, University of Cape

Coast, Ghana

\begin{abstract}
The low rate of screening for hepatitis B virus (HBV) in pregnant women is a highrisk factor for its vertical transmission. The objectives of this study were: i) to screen pregnant women for HBV infection; ii) vaccinate all children from birth against HBV regardless their mother HBV status; and iii) evaluate after 7 months of birth the level of their AbHBs among babies who received $\mathrm{HBV}$ vaccine at birth. Serological markers of HBV (HBsAg, HBeAg, AbHBs, AbHBe, and $\mathrm{AbHBc}$ ) were determined on venous blood samples from 237 pregnant women and their children using the Abon Biopharm Kit. One hundred and two (102) children received the three doses of the EUVAX $B{ }^{\circledR}$ vaccine respectively at birth, two months and four months of life. Seven months after
\end{abstract}

delivery, venous blood samples were collected from mothers and their children. Antibodies against hepatitis B surface antigen (AbHBs) were measured in vaccinated children using the ELISA Kit $A b H B S$ Quantitative EIA. DNA extraction was performed on samples from $\mathrm{HBV}$-seropositive mothers and their children using the Ribo Virus (HBV Real-TM Qual) Kit and for Real Time PCR, the HBV Real-TM Qual Kit was used. Serological diagnosis in pregnant women revealed $22(9.28 \%)$ hepatitis B surface antigen (HBsAg) positive samples of which 21 were positive for viral DNA by real-time PCR. Among the $22 \mathrm{HBsAg}+$ women, five (05) transmitted the virus to their children with a vertical transmission rate of $22.73 \%$. A transmission rate of $23.81 \%(5 / 21)$ was found with the PCR method. Analysis of AbHBs levels revealed that $98.31 \%$ of the children had an average concentration of $218.07 \pm 74.66 \mathrm{IU} / \mathrm{L}$, which is well above the minimum threshold for protection (11 IU/L). This study has confirmed that vertical transmission of $\mathrm{HBV}$ is a reality in Burkina Faso and that vaccination at birth would significantly reduce this transmission.

\section{Introduction}

Currently, the World Health Organization (WHO) estimates that 257 million people live with hepatitis $B$ virus (HBV) infection worldwide. ${ }^{1} \mathrm{HBV}$ is responsible for more than 686,000 deaths per year and this infection is considered as the second most common cause of cancer deaths after tobacco. ${ }^{1}$ Africa is the most affected region of the world with approximately 150 million cases of chronic hepatitis $\mathrm{B}$ and $\mathrm{C}$ associated with the risk of developing cirrhosis or liver cancer. $^{2}$ Burkina Faso (BF) which is a $\mathrm{HBV}$ high endemic area according to WHO classification has prevalence well above $8 \% .^{3-6}$

A study carried out at the University Hospital Yalgado Ouedraogo of Ouagadougou (CHU-YO) showed a prevalence of $4.1 \%$ in newborns to HBV infected mothers with a vertical transmission rate of $37.1 \% .^{7}$ Another study conducted in 2002 at the Saint Camille Hospital of Ouagadougou found a vertical transmission rate of $25.0 \%{ }^{8}$ Thus, according to McMahon et al. ${ }^{9}$ vertical transmission remains the most important mechanism of $\mathrm{HBV}$ chronic infections in Africa. The age at first exposure to HBV plays a very important role in the evolution of infection. Children born to mothers with active hepatitis $\mathrm{B}$, who are positive for $\mathrm{HBV}$ "e" antigen ( $\mathrm{HBeAg}+$ ), are at high risk of infection at birth or dur-
Correspondence: Jacques Simpore, Laboratory of Molecular Biology and Genetics (LABIOGENE), Department of Biochemistry and Microbiology, University Ouaga I Prof Joseph KI-ZERBO, BP 7021 Ouagadougou 03, Burkina Faso.

Tel.: +226-70230792

E-mail: jacques.simpore@yahoo.fr

Key words: Viral Hepatitis B, HBsAg, RealTime PCR, Vertical Transmission, Vaccination.

Acknowledgments: the authors would like to thank University Tor Vergata (Rome, Italy), Saint Camille Hospital and CERBA/LABIOGENE (Burkina Faso) for their support.

Contributions: ETY, data collection and manuscript preparation; ATY, BD, AKO, MA, data analysis and interpretation; $\mathrm{AKO}, \mathrm{BD}, \mathrm{TMZ}$, STS, IT, screening; FD, CS, CWMN, AG, vaccination; $\mathrm{CM}, \mathrm{PO}, \mathrm{DO}, \mathrm{VP}$; revision of the manuscript; JP, VC, conception and design and final approbation of the manuscript.

Conflict of interests: The authors declare no potential conflict of interest.

Funding: none.

Received for publication: 13 November 2017. Accepted for publication: 9 August 2018.

This work is licensed under a Creative Commons Attribution NonCommercial 4.0 License (CC BY-NC 4.0).

(C) Copyright E.T. Yelemkoure et al., 2018 Licensee PAGEPress, Italy

Journal of Public Health in Africa 2018; 9:816 doi:10.4081/jphia.2018.816

ing early childhood and of becoming chronic carriers. ${ }^{10}$ Moreover, the infection could occur during childhood and would imply transmission though breastfeeding. In addition, since the hepatitis B virus can be transmitted by blood, saliva and other fluids of the infected human organism, infected parents could transmit the virus to their infants and children as they take care of them including some traditional methods of caring for insert bites by applying saliva to site sites. If the latter transmission route plays a key role in HBV infection, then the local density of these blood-sucking arthropods would be a relevant factor to take into account during awareness against hepatitis B infection. ${ }^{3}$

Following this infection, approximately $25 \%$ of these children are at greater risk of death because they may develop hepatocellular carcinoma or cirrhosis in adulthood. ${ }^{9}$ The low rate of screening for HBV in preg- 
nant women is therefore a high risk factor for vertical transmission. Hence, the prevention of mother-to-child transmission of HBV plays a key role in the reduction of the disease prevalence. In many African countries, there is no active program to prevent mother-to-child transmission (PMTCT) of HBV. For a number of years, to prevent hepatitis $\mathrm{B}$ virus infection in children, the Ministry of Health of Burkina Faso, through the Expanded Program of Immunization (EPI), advocated the vaccine against HBV for all children at eight weeks of birth. This is already a strong prevention program but it would be necessary to vaccinate children from birth to block vertical transmission of the virus. Efforts to reduce significantly not only HBV infection in the general population but also its vertical transmission remain one of the global priorities in the fight against this public health disease.

Vaccination efforts have already been made: Collenberg et al. ${ }^{11}$ had observed AbHBs levels of $69.6 \%$ and $76.4 \%$ respectively in Nouna and Ouagadougou. Some studies have shown that immunoprophylaxis through hepatitis B vaccine with or without Hepatitis B Immunoglobulin G (HBIG) is effective in preventing the transmission of HBV infection to infants: Milne et al. ${ }^{12}$ ( seroprotection rates $=98.0-98.6 \%$ ); Zhu et al..$^{13}$ (seroprotection rates $=93.61 \%$ ); van den Ende et al. ${ }^{14}$ (seroprotection rates $\geq 96.0 \%$ ). A Brazilian study has also shown that administration of the hepatitis $B$ vaccine at birth is immunogenic with a good safety profile in newborns. ${ }^{15}$ As in this Brazilian study, and according to the transmission modes of HBV, it would be desirable to vaccinate newborns at birth to obtain data on the immunoprophylaxis of hepatitis $B$ vaccine in Burkina Faso.

The main objective of this research, which was to prevent mother-to-child transmission of hepatitis B virus in Burkina Faso, has three specific objectives: i) to screen pregnant women for HBV infection; ii) vaccinate all children at birth against HBV regardless their mother HBV status; and iii) evaluate after 7 months of birth the level of their AbHBs among babies who received $\mathrm{HBV}$ vaccine at birth.

\section{Materials and Methods}

\section{Type and period of study}

This is a descriptive cross-sectional study, which took place from September 2013 to June 2014 at Saint Camille Hospital of Ouagadougou (HOSCO).

\section{Sampling}

The study involved 237 term pregnant women and their newborns. All study participants gave their free written and informed consent according to the Helsinki Declarations. Four milliliters of venous blood were collected from pregnant women for HBV screening. Seven months after delivery, venous blood samples were collected from 102 children previously vaccinated against HBV. These samples were used to evaluate AbHBs titer serological tests and confirmation using real time PCR in children born to HBV positive mothers.

\section{HBV screening}

The HBV Combo Rapid Test Cassette Kit (Ref. No: IHBSG-401 Lot No.: HBSG14120001) was used for the rapid diagnosis of HBsAg and other markers of hepatitis B (HBeAg; AbHBe, AbHBs and $\mathrm{AbHBc}$ ) were then determined in $\mathrm{HBsAg}$ positive women.

\section{Newborns vaccination}

One hundred and two (102) newborns received the first dose of the HBV vaccine (EUVAX-B ${ }^{\circledR}$ Pediatric) at birth, followed by the second and third doses at two and four months respectively. All vaccinated children were regularly followed up throughout the study period to evaluate side effects and other adverse reactions.

\section{Assay for antibody titer}

The AbHBs titer was determined in children using the ELISA "AbHBs Quantitative EIA" Kit. It was obtained by averaging the concentration of three assays for each sample.

\section{Molecular diagnosis of $\mathbf{H B V}$}

Extraction of HBV DNA was performed using the "Genomic DNA Express" kit (Sacace Biotechnologies, Como, Italy). The HBV DNA was amplified by using the HBV Real-TM Qual Kit on the SaCycler-96
Real-Time PCR v.7.3 (Sacace Biotechnology, Como, Italy).

\section{Statistical analysis}

The data were analyzed using SPSS software version 17.0 (SPSS, Chicago, IL, USA) and Epi info version 7.0. The chisquare test was used for comparisons and the difference was considered statistically significant for $\mathrm{P}<0.05$.

\section{Ethical consideration}

Approval for this study was obtained from the Ethics Committee on Health Research of Burkina Faso (Deliberation No. 2013-7-065 on July 11, 2013). Written informed consent was obtained from all participants.

\section{Results}

HBV status according to sociodemographic characteristics of pregnant women

According to the data in Table 1, the women in this study were mostly housewives (50.2\%) with an average age of $27.0 \pm 5.3$ years. Among the 237 pregnant women enrolled, 22 were positive for HBsAg or a prevalence of $9.3 \%$. There was no statistically significant difference between the mean age of HBV seropositive $(28.1 \pm 6.7$ years) and seronegative (26.9 \pm 5.1 years) women ( $\mathrm{P}=0.309$, Table 1).

\section{Molecular diagnosis of HBsAg+ mothers and their children, and ver- tical transmission of $\mathrm{HBV}$}

Among the 22 pregnant women positive for HBsAg, 21 were confirmed positive for viral hepatitis B by PCR (HBV DNA + ) with a concordance rate of $95.4 \%(21 / 22)$.

Table 1. HBV status according to socio-demographic characteristics of pregnant women.

\begin{tabular}{lcccc} 
Pregnant women & $\mathbf{N}$ & HBV & HBV + & P-value \\
Housewives & 119 & 110 & 9 & 0.001 \\
Private sector & $50.2 \%$ & $46.4 \%$ & $3.8 \%$ & \\
& 42 & 33 & 9 & - \\
Schoolchildren/Students & $17.7 \%$ & $13.9 \%$ & $3.8 \%$ & \\
& 41 & 39 & 2 & - \\
Salaried employee & $17.3 \%$ & $16.5 \%$ & $0.8 \%$ & \\
& 35 & 33 & 2 & - \\
Total & $14.8 \%$ & $13.9 \%$ & $0.8 \%$ & \\
& 237 & 215 & 22 & 0.001 \\
Mean age (years) & & $90.7 \%$ & $9.3 \%$ & \\
& 237 & 215 & 22 & 0.390 \\
\hline
\end{tabular}


Table 2. HBV PCR results in HBsAg+ mothers and their children (Seven months after delivery) and Vertical Transmission of HBV.

\begin{tabular}{lllllllll} 
HBV-positive mothersChildren & & & & & & \\
Number of mothers & HBV DNA & HBsAg & HBeAg & AbHBs & AbHBe & AbHBc & HBV DNA & HBV vertical transmission \\
1 & + & + & + & - & - & + & + & 1 \\
4 & + & + & - & - & + & + & + & 4 \\
11 & + & + & - & - & + & + & - & 0 \\
4 & + & + & - & - & - & + & - & 0 \\
1 & - & + & - & - & - & - & - & 5 \\
22 & & & & & & & & \\
\hline
\end{tabular}

Table 3. The rate of AbHBs in vaccinated children seven months after delivery.

\begin{tabular}{lccc}
\hline AbHBs concentration (IU/L) & $N$ & AbHBs titer in children (UI/L) & Immune status \\
$X^{<} 11^{*}$ & $4 / 102$ & $5.4 \pm 4.1$ & Non-immune, $3.9 \%$ \\
$X^{>} 11$ & $98 / 102$ & $227.1 \pm 61.5$ & Immune, $96.1 \%$ \\
\hline Total & 102 & $218.1 \pm 74.7$ & \\
\hline
\end{tabular}

X: concentration; * t-TEST: $\mathrm{P}^{<} 0.001$

Among these $21 \mathrm{HBV}$ DNA+ women, five (5) transmitted the infection to their children with a vertical transmission rate of 23.81\% (5/21) (Table 2). These HBV-positive women have been referred to the specialist for medical care.

\section{Determination of anti-HBs antibody level in vaccinated children}

The average concentration of $\mathrm{HBsAg}$ from the 102 children was $218.1 \pm 74.7 \mathrm{IU} / \mathrm{L}$. A total of $98 / 102$ or $96.1 \%$ of children were immunized with an average AbHBs concentration above $11 \mathrm{IU} / \mathrm{L}(227.1 \pm 61.5 \mathrm{IU} / \mathrm{L})$ compared to $4 / 102$ or $3.9 \%$ of non-immunized with an average concentration of AbHBs less than $11 \mathrm{IU} / \mathrm{L}(5.4 \pm 4.1 \mathrm{IU} / \mathrm{L})$ (Table 3).

\section{Discussion}

In this study, $9.3 \%(22 / 237)$ of pregnant women were HBsAg positive. This prevalence is similar to the $9.3 \%$ and $9.8 \%$ reported by Simpore et al., ${ }^{3,4}$ respectively in 2004 and 2006 among pregnant women in Burkina Faso. However, it is lower than $12.04 \%$ and $11.4 \%$ respectively found by Ilboudo et al., ${ }^{8}$ and Sangare et al., ${ }^{7}$ in Ouagadougou and higher than those reported by Rouet et al. ${ }^{16}$ in Ivory Coast $(9.0 \%)$ and Maclean et al., ${ }^{17}$ in Northern Mali (8\%) among pregnant women. These results confirm the high endemicity of hepatitis B in the West African sub-region. This difference in the prevalence could probably be due to the samples size and especially the various distribution of HBV by countries and geographical areas. For example, the low rate of screening for $\mathrm{HBV}$ in pregnant women is a major risk factor for vertical transmission in sub-Saharan Africa countries.

Real-time PCR was performed to investigate the presence of viral DNA in the 22 HBsAg-positive mothers and their children. Twenty-one (21) mothers and five (05) children had HBV DNA corresponding to a vertical transmission rate of $23.8 \%(5 / 21)$.

The rate of $\mathrm{HBeAg}$ was $4.5 \%$ among mothers who transmitted the virus to their children. This rate is lower than $18.2 \%$, $11.1 \%$ and $31.4 \%$ respectively found by Nacro et al. ${ }^{18}$ in Bobo Dioulasso, Ilboudo et $a l .{ }^{8}$ and Sangare et al., ${ }^{7}$ in Ouagadougou, Burkina Faso. Indeed, previous studies reported that the presence of $\mathrm{HBeAg}$ in a pregnant woman is a significant risk factor for vertical transmission of HBV with a probability of $90 \%$ that the child become a chronic carrier.9,19 Mother-to-child transmission routes of HBV vary by geographic area. ${ }^{20}$ In this study, mothers who transmitted HBV to their children were positive for $\mathrm{HBsAg}, \mathrm{AbHBc}$, viral DNA, or $\mathrm{HBeAg}$. Indeed, studies conducted by Walz et al., ${ }^{21}$ and Ilboudo et al., ${ }^{22}$ showed that vertical transmission was possible for a mother positive for $\mathrm{AbHBc}, \mathrm{HBeAg}$ and viral DNA, respectively. These factors could explain the vertical transmission of HBV in the present study. Our results suggest a high risk of vertical transmission when pregnant woman is positive for $\mathrm{HBsAg}, \mathrm{AbHBc}$, $\mathrm{HBe} A g$, and viral DNA in sub-Saharan Africa, mainly in Burkina Faso.

Combined intramuscular hepatitis B vaccines have been studied in different countries. ${ }^{23,24}$ The immunogenicity of the three doses of EUVAX-B ${ }^{\circledR}$ intramuscular vaccine has already been confirmed in a previous study with protection rate of $89.5 \%{ }^{25}$ However, the present study is one of the first to demonstrate the immunogenicity and safety of intramuscular vaccine against hepatitis B at birth in Burkina Faso. These results from the three-dose series of primary hepatitis B vaccination from birth showed an immunization rate of $98.3 \%$ (AbHBs $>11 \mathrm{IU} / \mathrm{mL}$ ) in children seven months after vaccination. No adverse effects were reported in children throughout the study period. These results are consistent with those reported by Lee et al. ${ }^{26}$ and especially Carniel et al., ${ }^{15}$ in Brazil who have shown the safety and immunogenicity of this kind of vaccination in newborns.

These results could contribute to the development of a new kind of neonatal vaccine co-formulation algorithm to prevent significantly HBV, BCG, and Poliomyelitis virus infections in neonates. However, the lack of routine screening of pregnant women, incomplete vaccination coverage or delayed vaccine delivery are among other major risks to consider in reducing vertical transmission of $\mathrm{HBV}$.

\section{Conclusions}

Vaccination remains a major tool for preventing and controlling the transmission of the hepatitis B virus. This study confirms that vertical transmission of HBV is health issues in our country and that vaccination from birth would significantly reduce this transmission. These results could contribute to the development of a new strategy in the Expanded Program on Immunization (EPI) to prevent significantly the vertical transmission of HBV infection in Burkina Faso. 


\section{References}

1. WHO. Fact sheet $N^{\circ} 204$, Reviewed July 2017. World Health Organisation 2017. Available from: http://www.who. int/mediacentre/factsheets/fs204/en/ Accessed: August 2012.

2. Lutte contre les hépatites en Afrique. CONSENSUS de Dakar 2013. Available from: http://anima.blog. lemonde.fr/2013/07/27/lutte-contre-leshepatites-en-afrique-consensus-dedakar/ Accessed: September 2014.

3. Simpore J, Granato M, Santarelli R, et al. Prevalence of infection by HHV-8, HIV, HCV and HBV among pregnant women in Burkina Faso. J Clin Virol 2004;31:78-80.

4. Simpore J, Savadogo A, Ilboudo D, et al. Toxoplasma gondii, HCV, and HBV seroprevalence and co-infection among HIV-positive and -negative pregnant women in Burkina Faso. J Med Virol 2006;78:730-3.

5. Tao I, Compaore TR, Diarra B, et al. Seroepidemiology of Hepatitis B and C Viruses in the General Population of Burkina Faso. Hepatitis Res Treat 2014;2014:5.

6. Diarra B, Ouattara AK, Wendkuuni Djigma F, et al. World Hepatitis Day in Burkina Faso, 2016: Awareness, Screening, Identification of $\mathrm{HBV}$ Markers, HBV/HCV Coinfection, and Vaccination. Hepatitis Monthly 2017;17:e13789.

7. Sangaré L, Sombié R, Combasséré AW, et al. [Antenatal transmission of hepatitis $B$ virus in an area of HIV moderate prevalence, Burkina Faso]. Bull Soc Pathol Exot. 2009;102:226-9.

8. Ilboudo D, Sawadogo A, Simpore J. [Mother-to-child transmission of hepatitis B virus, in Ouagadougou, Burkina Faso]. Med Trop (Mars) 2002;62:99-101.

9. McMahon BJ. Natural History of Chronic Hepatitis B. Clin Liver Dis 2010;14:381-96.

10. Chang MH. Hepatitis B virus infection.
Sem Fetal Neonat Med 2007;12:160-7.

11. Collenberg E, Ouedraogo T, Ganamé J, et al. Seroprevalence of six different viruses among pregnant women and blood donors in rural and urban Burkina Faso: A comparative analysis. J Med Virol 2006;78:683-92.

12. Milne A, West DJ, Chinh DV, et al. Field evaluation of the efficacy and immunogenicity of recombinant hepatitis B vaccine without HBIG in newborn Vietnamese infants. J Med Virol 2002;67:327-33.

13. Zhu FC, Sun KX, Pan HX, et al. The immunogenicity in healthy infants and efficiency to prevent mother to child transmission of Hepatitis B virus of a $10 \mu \mathrm{g}$ recombinant yeast-derived Hepatitis B vaccine (Hep-KSC). Vaccine 2016;34:2656-62.

14. Van Den Ende C, Marano C, Van Ahee A, et al. The immunogenicity and safety of GSK's recombinant hepatitis B vaccine in adults: a systematic review of 30 years of experience. Exp Rev Vacc 2017;16:811-32.

15. Carniel EF, Morcillo AM, Blotta MH, et al. Immunogenicity and safety of combined intradermal recombinant Hepatitis B with BCG vaccines at birth. Vaccine 2008;26:647-52.

16. Rouet F, Chaix ML, Inwoley A, et al. $\mathrm{HBV}$ and $\mathrm{HCV}$ prevalence and viraemia in HIV-positive and HIVnegative pregnant women in Abidjan, Côte d'Ivoire: The ANRS 1236 study. J Med Virol 2004;74:34-40.

17. MacLean B, Hess RF, Bonvillain E, et al. Seroprevalence of hepatitis B surface antigen among pregnant women attending the Hospital for Women \& Children in Koutiala, Mali. South Afr Med J 2012;102:47-9.

18. Nacro B, Dao B, Dahourou H, et al. [HBs antigen carrier state in pregnant women in Bobo Dioulasso (Burkina Faso)]. Dakar Med 2000;45:188-90.

19. Pande C, Sarin SK, Patra S, et al. Hepatitis B vaccination with or without hepatitis B immunoglobulin at birth to babies born of HBsAg-positive mothers prevents overt HBV transmission but may not prevent occult $\mathrm{HBV}$ infection in babies: a randomized controlled trial. J Viral Hepat 2013;20:801-10.

20. Ranger-Rogez S, Denis Fo. Hepatitis B mother-to-child transmission. Exp Rev Anti-infect Ther 2004;2:133-45.

21. Walz A, Wirth S, Hucke J, Gerner P. Vertical Transmission of Hepatitis B Virus (HBV) from Mothers Negative for HBV Surface Antigen and Positive for Antibody to HBV Core Antigen. J Infect Dis 2009;200:1227-31.

22. Ilboudo D, Simpore J, Ouermi D, et al. Towards the complete eradication of mother-to-child HIV/HBV coinfection at Saint Camille Medical Centre in Burkina Faso, Africa. Braz J Infect Dis 2010;14:219-24.

23. Gabutti G, Bona G, Dentico P, et al. Immunogenicity and Reactogenicity following Primary Immunisation with a Combined DTaP-HBV Vaccine and a Haemophilus influenzae Type B Vaccine Administered by Separate or Mixed Injection. Clin Drug Investig 2005;25.

24. Pichichero ME, Bernstein H, Blatter MM, et al. Immunogenicity and Safety of a Combination Diphtheria, Tetanus Toxoid, Acellular Pertussis, Hepatitis B, and Inactivated Poliovirus Vaccine Coadministered with a 7-Valent Pneumococcal Conjugate Vaccine and a Haemophilus Influenzae Type $b$ Conjugate Vaccine. J Pediatr 2007;151:43-9.

25. Tele CA, Martins RM, Lopes Cl, Dos santos carneiro MA, Souza KP, Yoshida CF. Immunogenicity of a recombinant hepatitis B vaccine (Euvax-B) in haemodialysis patients and staff. Eur $\mathrm{J}$ Epidemiol 2001;17:145-9.

26. Lee C, Gong Y, Brok J, et al. Effect of hepatitis $\mathrm{B}$ immunisation in newborn infants of mothers positive for hepatitis B surface antigen: systematic review and meta-analysis. BMJ 2006;332:32836. 